\title{
NOX2-generated oxidative stress is associated with severity of ultrasound liver steatosis in patients with non-alcoholic fatty liver disease
}

\author{
Maria Del Ben ${ }^{1}$, Licia Polimeni ${ }^{1}$, Roberto Carnevale ${ }^{1}$, Simona Bartimoccia ${ }^{1}$, Cristina Nocella ${ }^{1}$, Francesco Baratta ${ }^{1}$, \\ Lorenzo Loffredo ${ }^{1}$, Pasquale Pignatelli ${ }^{1}$, Francesco Violi ${ }^{1}$ and Francesco Angelico ${ }^{2,3^{*}}$
}

\begin{abstract}
Background: Chronic oxidative stress is one of the key mechanisms responsible for disease progression in non-alcoholic fatty liver disease. However, so far, few studies reported increased circulating levels of oxidative stress markers in patients with non-alcoholic fatty liver and no study has been performed with newer markers of systemic oxidative stress. The aim was to assess the relationship between urinary 8-iso-prostaglandin F2a and serum soluble NOX2-derived peptide and the severity of liver steatosis in subjects with non-alcoholic fatty liver.

Methods: The study was performed in 264 consecutive patients referred for suspected metabolic disease. Steatosis was defined according to Hamaguchi ultrasonographic criteria. Oxidative stress was assessed by urinary 8-iso- prostaglandin F2a and serum soluble NOX2-derived peptide levels.

Results: Patients with non-alcoholic fatty liver had higher $(p<0.001)$ mean values of urinary 8-iso-PGF2 $a$ and of serum soluble NOX2-derived peptide, alanine aminotransferase, Cytokeratin-18 and homeostasis model of insulin resistance and lower values of serum adiponectin as compared to those without. Prevalence of metabolic syndrome and of its clinical features was significantly higher in patients with non-alcoholic fatty liver. Same findings were also observed after the exclusion of obese subjects, or subjects with diabetes or with metabolic syndrome and in those not taking statin medication. In addition, the levels of urinary 8-iso-PGF2a were independent predictors of non-alcoholic fatty liver and a strong association of urinary 8-iso-PGF2a and of serum soluble NOX2-derived peptide with the severity of steatosis at ultrasound was also observed.

Conclusions: We demonstrated increased markers of oxidative stress in subjects with non-alcoholic fatty liver. Urinary 8-iso-PGF2a and serum soluble NOX2-derived peptide levels were independent from obesity, diabetes and metabolic syndrome and increased with the severity of liver steatosis at ultrasound.
\end{abstract}

Keywords: Oxidative stress, Non-alcoholic fatty liver, 8-iso-PGF2a, sNOX2-dp, Metabolic syndrome

\section{Background}

Nonalcoholic fatty liver disease (NAFLD) includes a wide spectrum of liver diseases ranging from simple fatty liver to non-alcoholic steatohepatitis (NASH), which may progress to fibrosis and even cirrhosis and hepatocellular carcinoma [1]. It represents the most common and emerging chronic liver disease worldwide [2]. NAFLD is strongly

\footnotetext{
* Correspondence: francesco.angelico@uniroma1.it

${ }^{2}$ Department of Public Health and Infectious Disease, Sapienza University,

Rome, Italy

${ }^{3}$ I Clinica Medica - Policlinico Umberto I, Viale del Policlinico 155, 00161

Rome, Italy

Full list of author information is available at the end of the article
}

associated with obesity, insulin resistance, hypertension, and dyslipidemia, and is now regarded as the liver manifestation of the metabolic syndrome (MetS). a cluster of metabolic and cardiovascular risk factors including systemic inflammation and oxidative stress [3-8].

Traditionally, according to the "two hit" theory, simple steatosis and NASH have been considered an histological continuum with increasing degrees of severity [9]. More recently, the 'multiple parallel hits' hypothesis' has been proposed, suggesting simple steatosis and NASH as two unrelated disorders $[10,11]$.

\section{C) Biomed Central}

(c) 2014 Del Ben et al.; licensee BioMed Central Ltd. This is an Open Access article distributed under the terms of the Creative Commons Attribution License (http://creativecommons.org/licenses/by/2.0), which permits unrestricted use, distribution, and reproduction in any medium, provided the original work is properly credited. 
Several lines of evidence suggest that chronic oxidative stress is one of the key mechanisms responsible for liver damage and disease progression in NAFLD [9]. In particular, according to the "two-hit" theory, oxidative stress is a major player triggering the progression of steatosis to NASH as the result of an imbalance between prooxidant and anti-oxidant chemicals that lead to liver cell damage. In fact, the increased production of reactive oxygen species (ROS) is known to cause lipid peroxidation, followed by inflammation, and activation of stellate cells leading to fibrogenesis. Therefore, although the mechanisms underlying disease progression remain poorly understood, a therapeutic strategy targeting oxidative stress reduction has been proposed and, based on the results of a single randomized controlled trial [12], supplementation with vitamin $\mathrm{E}$ has been suggested by recent AASLD guidelines for the treatment of NASH in non diabetic subjects [13].

So far, few studies reported increased circulating levels of oxidative stress markers in patients with NAFLD [14-19]. However, most studies contained small number of patients and no study was able to document the relationship between the extent of steatosis and systemic markers of oxidative stress [20]. Larger studies with newer markers of oxidative stress are required since routine blood oxidative stress tests are unreliable markers of hepatic steatosis and probably do not accurately reflect hepatic oxidative stress. In this study, to assess oxidative stress in vivo, we measured urinary 8-iso-prostaglandin F2 $\alpha$ (8-iso-PGF2 $\alpha$ ) and serum levels of soluble NOX2derived peptide (sNOX2-dp). Measurement of urinary 8 -iso-PGF $2 \alpha$ is widely accepted as reliable indicator of oxidative stress in vivo [21,22]. Soluble NOX2-dp is a marker of NOX2 activation by blood cells, which is a member of the NADPH oxidase family which plays an important role in ROS generation [23,24]. Elevated urinary 8-iso-PGF2 $\alpha$ and serum sNOX2-dp levels have been described in a number of chronic inflammatory and metabolic diseases [25-28].

Aim of the present study was to assess the relationship between urinary 8 -iso-PGF2 $\alpha$ and serum sNOX2-dp and the severity of liver steatosis in subjects with NAFLD in different clinical settings.

\section{Methods}

\section{Study patients}

The study has been performed in 264 consecutive patients referred to our metabolic outpatient clinic for suspected metabolic disease, who had a liver ultrasonographic scanning (US) performed as part of routine clinical examination. To be eligible for the study, patients had to have fulfilled the following criteria: no history of current or past excessive alcohol drinking as defined by an average daily consumption of alcohol $>20 \mathrm{~g}$; negative tests for the presence of hepatitis B surface antigen and antibody to hepatitis $C$ virus; absence of history and clinical, biochemical and US findings consistent with cirrhosis and other chronic liver diseases. None of the subjects were taking amiodarone and other drugs known to promote fatty liver disease. Subjects underwent routine clinical and biochemical evaluation. Waist circumference, height and weight were recorded and body mass index (BMI) was calculated as weight $(\mathrm{Kg})$ divided by height $\left(\mathrm{m}^{2}\right)$. Blood pressure was recorded following standard procedures. Diabetes was diagnosed according to the WHO criteria [29]. Subjects taking insulin or oral antidiabetic drugs were considered to have diabetes. According to the modified criteria of the ATP III Expert Panel of the US National Cholesterol Education Program [30], MetS was diagnosed on the concomitant presence of at least three of the following five clinical features: waist circumference (central obesity) $>102 \mathrm{~cm}$ in men and $>88 \mathrm{~cm}$ in women, fasting blood glucose $\geq 100 \mathrm{mg} / \mathrm{dl}$, triglycerides $\geq 150 \mathrm{mg} / \mathrm{dl}$, HDL-cholesterol $<40 \mathrm{mg} / \mathrm{dl}$ in men and $<50 \mathrm{mg} / \mathrm{dl}$ in women, arterial systolic/diastolic blood pressure $\geq 130 / \geq$ $85 \mathrm{~mm} / \mathrm{Hg}$. A metabolic score was calculated for each patient based on the number of the discrete components of MetS identified. Written informed consent was obtained from all patients before the study. The study was approved by the ethics committee of the Policlinico Umberto 1 Hospital of Rome and conforms to the ethical guidelines of the 1975 Declaration of Helsinki.

\section{Laboratory measurements}

A venous blood sample and a spot urine sample were collected after a 10-14-h overnight fast. Serum total cholesterol, HDL-cholesterol and triglycerides were measured by an Olympus AN 560 apparatus using an enzymatic colorimetric method. LDL-cholesterol levels were calculated according to the Friedwald formula. Plasma insulin levels were assayed by commercially available radioimmunoassay. The homeostasis model of insulin resistance (HOMA-IR), based on serum fasting glucose and insulin levels, was used as a measure of insulin resistance [31]. Urinary 8-iso-prostaglandin F2 $\alpha$ (8-iso-PGF $2 \alpha$ ), as marker of whole body oxidative stress, was measured by a previously described and validated enzyme immunoassay method [32]. Intra-assay and interassay coefficients of variation were $2.1 \%$ and $4.5 \%$, respectively. Serum levels of soluble NOX2-derived peptide (sNOX2-dp) were detected by ELISA method as previously described [33]; intra-assay and inter-assay coefficients of variation were $5.2 \%$ and $6 \%$, respectively. Values are expressed as $\mathrm{pg} / \mathrm{ml}$. Adiponectin (APN) serum levels were measured with a commercial immunoassay (TemaRicerca, Italy). Intra-assay and inter-assay coefficients of variation were 6 and $8 \%$, respectively. Serum levels of Cytokeratin 18-M30 (CK-18) were measured as 
marker of liver damage with a commercial immunoassay (Tema Ricerca, Italy) and expressed as $\mathrm{mlU} / \mathrm{ml}$. Intra-assay and inter-assay coefficients were $6 \%$ and $7 \%$ respectively.

\section{Ultrasonographic examination}

Liver US scanning was performed to assess the degree of steatosis. All US were performed by the same operator who was blinded to laboratory values using an Esaote Medica apparatus equipped with a convex 3,5 MHz probe. Liver steatosis was defined according to Hamaguchi criteria based on the presence of abnormally intense, high level echoes arising from the hepatic parenchyma, liverkidney difference in echo amplitude, echo penetration into deep portion of the liver and clarity of liver blood vessel structure [34]. Steatosis was assessed semi-quantitatively on a scale of 0-6: 0 , absent; 1,2 mild; 3,4 moderate; 5,6 severe.

\section{Statistical analysis}

Statistical analysis was performed by using the SPSS statistical software version 8.0 for Windows (SPSS, Inc., Chicago. Illinois). Student's t-test for unpaired data was used for the comparison of mean values. Distribution of continuous variables was tested for normality using the a Kolmogorov-Smirnov test. Data are expressed as the mean \pm SD for normally distributed variables and as median followed by $25^{\text {th }}$ and $75^{\text {th }}$ centiles for non-normally distributed data. Group comparisons for normally distributed variables was performed by use of analysis of variance (ANOVA) and unpaired Student's t-test when appropriate, Non normally distributed variables were tested by Mann-Whitney test and Kruskall-Wallis test. Proportions and categorical variables were tested by the $X^{2}$-test and by the 2-tailed Fisher's exact method when appropriate. All $P$ values are two-tailed; a $P$ value of less than 0.05 was considered to indicate statistical significance. Multiple linear regression analyses and a stepwise logistic regression analysis testing for the dichotomous response variable presence or absence of NAFLD were performed after controlling for possible clinical and biochemical confounders. The predictor variables entered in the different regression models were age, gender, BMI, diabetes, MetS, HOMA-IR, serum triglycerides, adiponectin, cytokeratin-18, urinary 8 -iso-PGF2 $\alpha$ and statin use.

\section{Results}

Table 1 reports some clinical and biochemical characteristics of subjects with and without NAFLD. Patients with NAFLD had significantly higher $(\mathrm{p}<0001)$ mean values of urinary 8-iso-PGF2 $\alpha$ and of serum sNOX2-dp, ALT, CK18 and HOMA-IR and lower values of serum adiponectin.
Table 1 Clinical and biochemical characteristics of subjects with and without NAFLD

\begin{tabular}{lccc}
\hline & $\begin{array}{c}\text { NAFLD } \\
(\mathbf{2 1 3})\end{array}$ & $\begin{array}{c}\text { w/o NAFLD } \\
\mathbf{( 5 1 )}\end{array}$ & p \\
\hline Age (yrs) & $54,3 \pm 12$ & $56,1 \pm 14,4$ & Ns \\
Male Gender (\%) & 64,6 & 63,3 & Ns \\
BMI (kg/m ${ }^{2}$ ) & $31,6 \pm 5,6$ & $26,8 \pm 3,6$ & $<0,001$ \\
Urinary 8-iso-PGF2a (pg/mg & $714,4 \pm 121,5$ & $621,2 \pm 125,9$ & $<0,001$ \\
creatinine) & & & \\
sNOX2-dp (pg/ml) & $57,4 \pm 13.6$ & $47,8 \pm 9.9$ & $<0.001$ \\
Adiponectin (ng/ml) & $8,5(5 / 12)$ & $13(8 / 15)$ & $<0,001$ \\
Cytokeratin 18 (mIU/ml) & $180(146 / 190)$ & $136(125 / 173)$ & $<0,001$ \\
ALT (IU/L) & $27,5(20 / 40)$ & $18(14 / 27)$ & $<0,001$ \\
HOMA_IR & $3,5(2,4 / 5,9)$ & $1,9(1,3 / 2,3)$ & $<0,001$ \\
Metabolic syndrome (\%)* & 67,7 & 21,7 & $<0,001$ \\
High fasting glucose (\%)* & 53,8 & 29,8 & $<0,01$ \\
Hypertriglyceridemia (\%)* & 46,9 & 14,6 & $<0,001$ \\
High waist circumference (\%)* & 81,9 & 42,6 & $<0,001$ \\
Low HDL-cholesterol (\%)* & 37,2 & 18,8 & $<0,05$ \\
High blood pressure (\%)* & 84,5 & 75,5 & Ns \\
Diabetes (\%) & 31,1 & 12,2 & $<0,01$ \\
Statin use (\%) & 33,5 & 42,9 & Ns \\
\hline
\end{tabular}

*According to ATPIII modified criteria (Ref. 30).

Prevalence of MetS and of most of its clinical features was significantly higher in patients with NAFLD.

Bivariate correlation coefficients between the study variables are reported in Table 2. A strong positive correlation was found between Urinary 8-iso-PGF2 $\alpha$ and serum sNOX2-dp $(\mathrm{r}=0.745 ; \mathrm{p}<0.001)$. Both variables were positively correlated $(\mathrm{p}<0.001)$ with Hamaguchi and MetS scores, BMI, serum CK-18 and HOMA-IR and negatively correlated $(\mathrm{p}<0.001)$ with serum adiponectin.

In order to better evaluate the independent effect of NAFLD on the above variables, separate comparisons between subjects with and without NAFLD were performed in subgroups of subjects.

without diabetes, MetS, obesity and statin use (Table 3). In all subgroups, mean urinary 8 -iso-PGF2 $\alpha$ and serum sNOX2-dp were significantly higher in subjects with NAFLD, as compared with those without NAFLD. Moreover, most of the other differences were still statistically significant when comparisons were performed in subjects without diabetes, without obesity, without MetS and in those not taking statins. Prevalence of NAFLD and of severe steatosis significantly increased in the increasing tertiles of urinary 8 -iso-PGF2 $\alpha$ (66.3 vs 83.7 vs 91.9; $\mathrm{p}<0.001$ and 13.8 vs 30.3 vs 45.6 : $\mathrm{p}<0.001$, respectively).

Table 4 reports mean values of some biochemical variables according to NAFLD severity at ultrasound examination. A progressive, statistically significant increase $(p<.001)$ of mean values of urinary 8 -iso-PGF2 $\alpha$ and 
Table 2 Linear correlation coefficients between some clinical and biochemical variables

\begin{tabular}{|c|c|c|c|c|c|c|c|c|c|}
\hline & PGF2a & & & & & & & & \\
\hline Urinary 8-iso-PGF2a (pg/mg creatinine) & 1 & BMI & & & & & & & \\
\hline Body mass index (kg/m2) &, $803^{* *}$ & 1 & Age & & & & & & \\
\hline Age (yrs) &,- 051 &,- 089 & 1 & Adipo & & & & & \\
\hline Adiponectin (ng/mL) &,$- 686^{* *}$ &,$- 838^{* *}$ &, 004 & 1 & CK-18 & & & & \\
\hline Cytokeratin-18 (mIU/ml) &, $684^{* *}$ &, $723^{* *}$ & 011 &,$- 602^{* *}$ & 1 & NOX2 & & & \\
\hline sNOX2-dp (pg/ml) &, $745^{* *}$ &, $570^{* *}$ &,- 074 &,$- 430^{* *}$ &, $491^{* *}$ & 1 & ALT & & \\
\hline $\mathrm{ALT}(\mathrm{IU} / \mathrm{L})$ & ,002 & 017 &,- 098 & ,031 & ,061 &,- 007 & 1 & HOMA-IR & \\
\hline HOMA-IR & $321^{* *}$ &, $417^{* *}$ &, 063 & $-299^{* *}$ & $354^{* *}$ &, $288^{* *}$ &, $254^{* *}$ & 1 & MetS code \\
\hline MetS code§ &, $390^{* *}$ &, $468^{* *}$ & $191^{* *}$ &,$- 405^{* *}$ &, $436^{* *}$ &, $351^{* *}$ &, $143^{*}$ &, $536^{* *}$ & 1 \\
\hline Hamaguchi score^^ &, $374^{* *}$ &, $477^{* *}$ & 012 &,$- 369^{* *}$ &, $339 * *$ &, $325^{* *}$ &, $416^{* *}$ &, $497^{* *}$ &, $481^{* *}$ \\
\hline
\end{tabular}

${ }^{*} \mathrm{p}<.01 ;{ }^{* *} \mathrm{p}<.001 ;{ }^{\varsigma}$ no. of variables of MetS according to ATPIII modified criteria (Ref. 30); $\wedge$ see Methods.

of serum sNOX2-dp, CK-18, ALT, gamma-glutamiltranspeptidase (GGT) and HOMA-IR was observed from the group without fatty liver to the groups with mild, moderate and severe steatosis, while a decreasing trend was observed for serum adiponectin.

Since urinary 8 -iso-PGF2 $\alpha$ and serum sNOX2-dp values were also significantly correlated with HOMA-IR and MetS score, a separate analysis was performed in patients with MetS. Also in this clinical setting, an increasing trend of the mean levels of both urinary 8-iso-PGF2 $\alpha$ and serum sNOX2-dp with increasing NAFLD severity was observed.

Table 5 reports the results of the stepwise multiple logistic regression analysis performed to assess the independent contribution of urinary 8-iso-PGF2 $\alpha$ for the prediction of NAFLD. Age, urinary 8-iso-PGF2 $\alpha$ and MetS were independent predictors of NAFLD, after controlling for gender, BMI, HOMA-IR, serum triglycerides and adiponectin, diabetes and statin use.

Two more regression analyses were performed to evaluate factors independently related to the severity of the NAFLD (serum cytokeratin-18 levels) and factors independently related to the degree of systemic oxidative stress (urinary 8-iso-PGF2 $\alpha$ ).

In the first multiple linear regression analysis, BMI (standardized coefficient $\beta=0.236 ; \mathrm{SE}=0.725 ; \mathrm{p}=0.019$ ), urinary 8 -iso-PGF $\alpha$ (standardized coefficient $\beta=0.268$; $\mathrm{SE}=0.029 ; \mathrm{p}=0.002$ ) and serum adiponectin (standardized coefficient $\beta=-0.193 ; \mathrm{SE}=0.726 ; \mathrm{p}=0.012$ ) were independent predictors of serum CK-18 levels $\left(R^{2}=0.39\right)$. In the second regression analysis, BMI (standardized coefficient $\beta=0.687 ; \mathrm{SE}=1.430 ; \mathrm{p}=0.000$ ) and serum cytokeratin-18 (standardized coefficient $\beta=2.904$; $\mathrm{SE}=$ $0.144 ; \mathrm{p}=0.004$ ) were independently associated with urinary 8-iso-PGF2 $\alpha$ levels $\left(R^{2}=0.66\right)$.

\section{Discussion}

Our findings show, for the first time, an increased systemic oxidative stress in subjects with NAFLD, as assessed by increased levels of urinary 8 -iso-PGF2 $\alpha$, currently regarded as the best measure of oxidative stress in vivo and of serum sNOX2-dp, a marker of NOX2 activation by blood cells, which plays an important role in ROS production [21-23].

So far, few studies have investigated markers of systemic oxidative stress in subjects with NAFLD. However, no study has documented the relationship between urinary 8-iso PGF2 $\alpha$ and serum sNOX2-dp and the extent of fatty liver. In a small study performed in 21 subjects with NASH and 19 controls, subjects with NASH had significantly higher levels of oxidized LDL and of thiobarbituric acid-reacting substances (TBARS) suggesting an increased cardiovascular risk [14]. Similar results were reported in India, where TBARS levels were significantly elevated and GSH/GSSG ratio was significantly decreased in NAFLD subjects without and with type 2 diabetes [14]. Increased systemic levels of malondialdehyde were observed in 58 male patients with histologically proven NAFLD compared to healthy age matched males [15]. In a further study, NAFLD children with immune responses against MDA derived antigens showed more severe lobular inflammation and had a 13-fold higher prevalence of overt NASH suggesting the presence of oxidative stress in a high proportion of NAFLD children [17]. In two more study the percentage of hepatocytes positive for 8-OHdG expression and serum 8-OHdG levels were significantly higher in patients with NASH than simple fatty liver, while the oxidative stress marker GGT was increased in both conditions $[18,35,36]$. Finally, in a recent cross-sectional study, oxidative stress detected as the ratio of plasma total antioxidant status to total oxidant status was associated with insulin resistance in obese adolescents with NAFLD [19].

Our study was carried out in a large sample of consecutive patients referred for suspected metabolic disease, who had a liver US performed as part of routine clinical examination. We found statistically significant 
Table 3 Clinical and biochemical characteristics in subjects without diabetes, or obesity or metabolic syndrome and in statin non users according to the presence or absence of NAFLD

\begin{tabular}{|c|c|c|c|c|c|c|c|c|}
\hline & \multicolumn{2}{|c|}{ Subjects w/o diabetes } & \multicolumn{2}{|c|}{ Subjects w/o obesity^ } & \multicolumn{2}{|c|}{ Subjects w/o MetS } & \multicolumn{2}{|c|}{ Subjects w/o statin use } \\
\hline & Nafld (144) & no-Nafld (43) & Nafld (85) & no-Nafld (39) & Nafld (73) & no-Nafld (41) & Nafld (139) & no-Nafld (28) \\
\hline Age (yrs) & $52,1 \pm 12,7$ & $54,4 \pm 14$ & $55,0 \pm 13,0$ & $55,0 \pm 14,7$ & $50,1 \pm 13,1$ & $54,7 \pm 13,0$ & $50,8 \pm 11,8$ & $53,0 \pm 15,9$ \\
\hline Male gender (\%) & 33,3 & 34,9 & 23,8 & 34,2 & 31,8 & 30,6 & 36,0 & 39,3 \\
\hline BMl & $30,9 \pm 5,6$ & $26,9 \pm 3,7 * * *$ & $26,6 \pm 2,3$ & $25,2 \pm 2,0^{* * *}$ & $29,5 \pm 5,7$ & $26,5 \pm 3,5^{* *}$ & $31,0 \pm 5,8$ & $27,2 \pm 3,9^{* * *}$ \\
\hline Urinary 8-iso-PGF2a (pg/mg creatinine) & $703,4 \pm 117,9$ & $634,1 \pm 106,7^{* * *}$ & $626,6 \pm 81,5$ & $583,6 \pm 102,7^{*}$ & $672,1 \pm 123,0$ & $619,3 \pm 95,1^{*}$ & $711,6 \pm 133$ & $627,5 \pm 147,9^{* *}$ \\
\hline sNOX2-dp (pg/ml) & $56,4 \pm 13,8$ & $49,9 \pm 8,9^{* * *}$ & $49,4 \pm 12,9$ & $45,8 \pm 8,9$ & $54,0 \pm 13,3$ & $48,4 \pm 8,3^{*}$ & $57,5 \pm 14,1$ & $47,5 \pm 10,7^{* * *}$ \\
\hline Adiponectin (ng/ml) & $8,5(5,5 / 12)$ & $13,5(7,5 / 15)^{* * *}$ & $12,5(10,5 / 14)$ & $13,5(12,5 / 14,5)^{* *}$ & $10,5(6,2 / 13,6)$ & $13,0(6,5 / 14)^{*}$ & $7,5(5 / 11,5)$ & $13,5(6,8 / 15)^{* * *}$ \\
\hline Cytokeratin-18 (mlU/ml) & $174,5(136 / 189)$ & $130,0(125 / 175)^{* *}$ & $149,0(125 / 179)$ & $126,0(110 / 165)^{*}$ & $161,5(125 / 180$ & $165,0(125 / 178)$ & $179,0(145 / 190)$ & $140,0(125 / 179)^{* *}$ \\
\hline ALT (IU/L) & $26(20 / 40)$ & $20(15 / 27)^{* * *}$ & $34(23 / 42)$ & $15(13 / 24)^{* * *}$ & $26(19 / 27)$ & $18(14 / 27)^{* *}$ & $27(20 / 40)$ & $18(14 / 27)^{* * *}$ \\
\hline GGT (IU/L) & $25(17 / 40)^{*}$ & $20(14 / 28)$ & $30(20 / 52)$ & $21(16 / 23)^{* *}$ & $23(16 / 10)$ & $21(18 / 36)$ & $25(17 / 44)^{*}$ & $21(13 / 30)$ \\
\hline HOMA_IR & $2,9(2,1 / 4,5)$ & $1,8(1,2 / 2,1)^{* * *}$ & $2,8(1,8 / 4,3)$ & $2,0(1,6 / 3,2)$ & $2,6(1,7 / 3,4)$ & $1,8(1,5 / 2,1)^{*}$ & $3,1(2,2 / 5,5)$ & $1,8(1,1 / 2,1)^{* * *}$ \\
\hline $\operatorname{Met}^{\S}(\%)$ & 57,0 & $12,5^{* * *}$ & 51,8 & $19,4^{* * *}$ & 0 & 0 & 57,8 & $16,0^{* * *}$ \\
\hline High Fasting Glucose ${ }^{\S}(\%)$ & 32,9 & 19,5 & 44,0 & 30,6 & 15,2 & 13,9 & 41,3 & 23,1 \\
\hline High Triglycerides $^{\S}(\%)$ & 44,8 & $11,9^{* * *}$ & 39,3 & $16,2^{*}$ & 7,6 & 13,9 & 40,9 & $11,1^{* *}$ \\
\hline High Waist Circumference ${ }^{\S}(\%)$ & 78,2 & $43,9^{* * *}$ & 55,4 & $27,0^{* *}$ & 59,1 & 38,9 & 80 & $46,2^{* * *}$ \\
\hline Low C-HDL ${ }^{\S}(\%)$ & 35,7 & $16,7^{*}$ & 31,0 & 21,6 & 6,1 & 13,9 & 37,2 & $14,8^{*}$ \\
\hline High Blood Pressure $\left.{ }^{\S} \%\right)$ & 79,0 & 74,4 & 81,0 & 73,7 & 69,7 & 75,0 & 80,3 & 75,0 \\
\hline Diabetes (\%) & 0 & 0 & 21,4 & 15,8 & 7,6 & 2,8 & 20,1 & 7,1 \\
\hline Statin use (\%) & 22,9 & $39,5^{*}$ & 31,0 & 47,4 & 13,6 & $41,7^{* *}$ & 0 & 0 \\
\hline
\end{tabular}


Table 4 Some biochemical variables in all subjects and in subjects with MetS according to NAFLD severity at ultrasound examination

\begin{tabular}{|c|c|c|c|c|c|c|c|c|c|c|}
\hline \multirow{3}{*}{ Variables } & \multicolumn{5}{|c|}{ ALL SUBJECTS } & \multicolumn{5}{|c|}{ SUBJECTS WITH MetS } \\
\hline & \multicolumn{10}{|c|}{$\overline{N A} \overline{F L D}$} \\
\hline & $\begin{array}{l}\text { Absent } \\
(n=51)\end{array}$ & $\begin{array}{c}\text { Mild } \\
(n=45)\end{array}$ & $\begin{array}{l}\text { Moderate } \\
(n=88)\end{array}$ & $\begin{array}{l}\text { Severe } \\
(n=80)\end{array}$ & $\mathbf{P}$ & $\begin{array}{l}\text { Absent } \\
(n=10)\end{array}$ & $\begin{array}{c}\text { Mild } \\
(n=21)\end{array}$ & $\begin{array}{c}\text { Moderate } \\
(n=54)\end{array}$ & $\begin{array}{l}\text { Severe } \\
(n=63)\end{array}$ & $\mathbf{P}$ \\
\hline $\begin{array}{l}\text { Urinary 8-iso-PGF2a } \\
\text { (pg/mg creatinine) }\end{array}$ & $\begin{array}{c}621,2 \pm \\
125,9\end{array}$ & $\begin{array}{c}674,3 \pm \\
121,2\end{array}$ & $\begin{array}{c}700,7 \pm \\
108,4\end{array}$ & $\begin{array}{c}751,3 \pm \\
121,0\end{array}$ & $<, 001$ & $\begin{array}{c}616,6 \pm \\
213,2\end{array}$ & $\begin{array}{c}700,2 \pm \\
103,9\end{array}$ & $\begin{array}{c}734,3 \pm \\
34,5\end{array}$ & $\begin{array}{c}761,9 \pm \\
96,5\end{array}$ & $<, 001$ \\
\hline sNOX2-dp (pg/ml) & $47,9 \pm 9,9$ & $52,9 \pm 15,5$ & $57,1 \pm 13.3$ & $60,0 \pm 12,2$ & $<.001$ & $45,3 \pm 14,2$ & $56,0 \pm 13,3$ & $58,9 \pm 12,6$ & $60,4 \pm 10.6$ & $<, 005$ \\
\hline $\begin{array}{l}\text { Adiponectin } \\
(\mathrm{ng} / \mathrm{ml})\end{array}$ & $\begin{array}{c}13,0 \\
(8,0 / 15,0)\end{array}$ & $\begin{array}{c}10,0 \\
(5,5 / 12,0)\end{array}$ & $8,8(6,0 / 13,5)$ & $6,0(4,9 / 9,0)$ & $<, 001$ & $\begin{array}{c}12,5 \\
(5,0 / 13,9)\end{array}$ & $9,4(5,8 / 11,8)$ & $7,5(5,0 / 11,1)$ & $6,0(4,6 / 8,5)$ & $<, 05$ \\
\hline $\begin{array}{l}\text { Cytokeratin } 18 \\
(\mathrm{mlU} / \mathrm{ml})\end{array}$ & $\begin{array}{c}136 \\
(125 / 173)\end{array}$ & $\begin{array}{c}169 \\
(129 / 185)\end{array}$ & $\begin{array}{c}176 \\
(140 / 190)\end{array}$ & $\begin{array}{c}180 \\
(168 / 182)\end{array}$ & $<, 001$ & $\begin{array}{c}147 \\
(128 / 182)\end{array}$ & $\begin{array}{c}170 \\
(132 / 188)\end{array}$ & $\begin{array}{c}180 \\
(162 / 190)\end{array}$ & $\begin{array}{c}182 \\
(175 / 195)\end{array}$ & $<, 05$ \\
\hline ALT (IU/L) & $18(14 / 27)$ & $21(17 / 34)$ & $26(19 / 36)$ & $34(25 / 45)$ & $<, 001$ & $16(12, / 22)$ & $26(19 / 37)$ & $25,5(17 / 36,3)$ & $32(24 / 43)$ & $<, 001$ \\
\hline GGT (IU/L) & $21(14 / 30,5)$ & $23(16 / 40,7)$ & $21(16 / 35)$ & $\begin{array}{l}33,50 \\
(24 / 61)\end{array}$ & $<, 001$ & $14(11 / 25)$ & $25(19 / 42)$ & $22(16 / 34)$ & $34(24 / 64)$ & $<, 001$ \\
\hline HOMA_IR & $1,9(1,3 / 2,3)$ & $2,5(1,7 / 3,8)$ & $3,3(2,2 / 5,4)$ & $5,2(3,1 / 7,3)$ & $<, 001$ & $2,9(1,1 / 3,8)$ & $2,9(1,7 / 4,5)$ & $3,9(2,9 / 5,9)$ & $5,6(3,3 / 7,4)$ & $<, 001$ \\
\hline $\begin{array}{l}\text { Metabolic } \\
\text { syndrome (\%)* }\end{array}$ & 21,7 & 50,0 & 63,5 & 81,8 & $<, 001$ & 100 & 100 & 100 & 100 & - \\
\hline
\end{tabular}

*Defined according to ATPIII modified criteria (Ref. 30).

higher urinary 8-iso-PGF2 $\alpha$ and serum sNOX2-dp levels in subjects with fatty liver, as compared to those without. The same findings were also observed after the exclusion of obese subjects, or subjects with diabetes or with MetS and in those not taking statin medication. In addition, the levels of urinary 8-iso-PGF $2 \alpha$ were independent predictors of NAFLD and a strong association of urinary 8 -iso-PGF2 $\alpha$ and of serum sNOX2-dp with the severity of liver steatosis at ultrasound examination was also observed. In our study, a correlation was also found between HOMA-IR, urinary 8-iso PGF2 $\alpha$ and sNOX2-dp, confirming the interdependency of insulin resistance and oxidative stress in the pathogenesis of NAFLD. Moreover, urinary 8 -iso-PGF2 $\alpha$ were also independent predictors of serum CK-18, a marker of apoptosis reflecting liver disease severity, further suggesting a possible role of oxidative stress in the progression from simple fatty liver to NASH [37].

Our results support the hypothesis that NAFLD is associated to enhanced oxidative stress as the result of increased 8-isoprostane production induced by NOX2 activation. Although the association between NAFLD and markers of increased oxidative stress does not necessary imply causality, our findings suggest oxidative stress as a possible target of antioxidant therapy in patients with NAFLD and give support to the indication for vitamin $\mathrm{E}$ supplementation for non diabetic, non cirrhotic patients with NASH $[12,13]$.

Measurement of 8 -iso-PGF2 $\alpha$ has overcome many of the limitations associated with other methods and has emerged as the most reliable approach to assess the role of in vivo oxidative stress status in the pathogenesis of human disease. Isoprostanes are stable prostaglandinlike compounds that are produced by free radical mediated lipid peroxidation in vivo are as a result of oxidative damage to cell membranes. They are unaffected by dietary lipid composition and are increased in animal models of oxidant injury. Based on these characteristics, F2isoprostanes have emerged as the gold standard of oxidative stress in vivo, especially if determined in urine $[22,23]$. In fact, measurement of urinary 8 -iso-PGF $2 \alpha$ has several advantages such as non invasiveness, stability and absence of any significant daily and day-to-day variability. We have previously demonstrated increased urinary 8-iso PGF $2 \alpha$ values also in other chronic conditions such as hypercholesterolemia, metabolic syndrome, obstructive sleep apnoea syndrome and peripheral artery disease [25-28]. Recently, women with high levels of

Table 5 Stepwise multiple logistic analysis of independent predictors of NAFLD in 264 subjects

\begin{tabular}{lcccccc}
\hline & B & S.E. & P & O.R. & \multicolumn{2}{c}{ 95,0\% C.I. for O.R. } \\
\hline Age (yrs) & & & & & Lower & Upper \\
Urinary 8-isoprostanes (pg/mg creatinine) &,- 048 &, 021 &, 021 &, 953 &, 915 & 1,993 \\
Metabolic syndrome &, 006 &, 002 &, 003 & 1,006 & 1,010 \\
\hline
\end{tabular}

Variables entered on step 1: age, gender, BMI, HOMA-IR, serum triglycerides and adiponectin, urinary 8-isoprostanes, metabolic syndrome, diabetes, statin use. 
urinary 8-iso PGF2 $\alpha$ were reported to have an $80 \%$ increased risk of dying of coronary heart disease or stroke, supporting the involvement of oxidative stress also in the pathophysiology of cardiovascular disease [36]. In our study, cytokeratin-18, a marker of liver disease severity, was an independent predictor of urinary 8-iso PGF2 $\alpha$, thus suggesting also a possible effect of fatty liver on systemic oxidative stress.

Our study may have some limitations. First, we detected fatty liver by ultrasound, which is a qualitative method inadequate to quantify less than $20 \%$ liver fat content [38]. Moreover, the Hamaguchi ultrasonographic score does not specifically predict NASH. The gold standard for the diagnosis of NASH is liver biopsy, but this is an invasive procedure with potentially serious complications and is therefore not acceptable without clinical indication. We acknowledge that grades of steatosis could have been better determined by magnetic resonance spectroscopy. However, Hamaguchi score showed $100 \%$ specificity and $91.7 \%$ sensitivity when compared with liver biopsy in NAFLD patients [34]. Second, although performed in a large series of patients, the study has been carried out in patients recruited in a Hospital-based setting. Finally, this is a cross-sectional study and it is therefore impossible to say whether oxidative stress, caused by obesity, contributes to the pathophysiology of NAFLD (or perhaps more NASH) or whether the inflamed liver contributes to the systemic oxidative stress.

\section{Conclusion}

We demonstrated increased markers of oxidative stress in subjects with NAFLD. Urinary 8-iso-PGF2 $\alpha$ and serum soluble NOX2-derived peptide levels were independent from obesity, diabetes and MetS and increased with the severity of liver steatosis at ultrasound. Our findings are consistent with the "two-hit" theory based on the prominent role of oxidative stress as a major player triggering the progression of steatosis to NASH. Moreover, they are also in agreement with the more recent "multiple parallel hits" theory including genetic predisposition, intestinal macrobiota and insulin resistance as the major contributors of increased oxidative stress and progressive liver damage [10]. Finally, they may also give support to the recommendation for early antioxidant treatment extended even to subjects with simple steatosis.

\section{Abbreviations}

NAFLD: Non-alcoholic fatty liver disease; NASH: Non-alcoholic steatohepatitis; 8-iso-PGF2a: 8-iso-prostaglandin F2a; sNOX2-dp: Soluble NOX2-derived peptide; ALT: Alanine aminotransferase; CK-18: Cytokeratin-18; HOMA-IR: Homeostasis model of insulin resistance; MetS: Metabolic syndrome; ROS: Reactive oxygen species; US: Ultrasonographic scanning; BMI: Body mass index; TBARS: Thiobarbituric acid-reacting substances; GGT: Gamma-glutamil-transpeptidase.

\section{Competing interests}

All authors declare that they have no competing interests.

\section{Authors' contributions}

MDB contributed to study design and wrote the manuscript. LP, RC, FB, SB and $C N$ contributed to data collection, analysis and interpretation; $L L$ and PP reviewed the manuscript; FV reviewed and edited the manuscript. FA designed the study and wrote the manuscript; he is the guarantor of this work and, as such, had full access to all the data in the study and takes responsibility for the integrity of the data and the accuracy of the data analysis. All Authors approved the final manuscript.

\section{Acknowledgments}

The Authors wish to thank nurses Monica Brancorsini and Daniela Salzano for their skillful cooperation.

\section{Author details}

${ }^{1}$ Department of Internal Medicine and Medical Specialties, Sapienza University, Rome, Italy. ${ }^{2}$ Department of Public Health and Infectious Disease, Sapienza University, Rome, Italy. ${ }^{3}$ I Clinica Medica - Policlinico Umberto I, Viale del Policlinico 155, 00161 Rome, Italy.

Received: 8 August 2013 Accepted: 16 April 2014

Published: 23 April 2014

\section{References}

1. Angulo P: Nonalcoholic fatty liver disease. N Engl J Med 2002, 346:1221-1231.

2. Bedogni G, Miglioli L, Masutti F, Tiribelli C, Marchesini G, Bellentani S: Prevalence of and risk factors for non-alcoholic fatty liver disease: the dionysos nutrition and liver study. Hepatology 2005, 42:44-52.

3. Angelico F, Del Ben M, Conti R, Francioso S, Feole K, Maccioni D, Antonini TM, Alessandri C: Non alcoholic fatty liver disease: an hepatic consequence of common metabolic diseases. J Gastroenterol Hepatol 2003, 18:588-594.

4. Kotronen A, Yki-Järvinen H: Fatty liver: a novel component of the metabolic syndrome. Arterioscler Thromb Vasc Biol 2008, 28:27-38.

5. Angelico F, Del Ben M, Conti R, Francioso S, Feole K, Fiorello S, Cavallo MG I, Zalunardo B, Lirussi F, Alessandri C, Violi F: Insulin resistance, the metabolic syndrome, and nonalcoholic fatty liver disease. J Clin Endocrinol Metab 2005, 90:1578-1582.

6. Targher G, Day CP, Bonora E: Risk of cardiovascular disease in patients with nonalcoholic fatty liver disease. N Engl J Med 2010, 363:1341-1350.

7. Del Ben M, Baratta F, Polimeni L, Angelico F: Non-alcoholic fatty liver disease and cardiovascular disease: epidemiological, clinical and pathophysiological evidences. Intern Emerg Med 2012, 7(Suppl 3):S291-S296.

8. Videla LA, Rodrigo R, Araya J, Poniachik J: Insulin resistance and oxidative stress interdependency in non-alcoholic fatty liver disease. Trends $\mathrm{Mol}$ Med 2006, 12:555-558.

9. Day CP: Pathogenesis of steatohepatitis. Best Pract Res Clin Gastroenterol 2002, 16:663-678.

10. Tilg H, Moschen AR: Evolution of inflammation in nonalcoholic fatty liver disease: the multiple parallel hits hypothesis. Hepatology 2010, 52:1836-1846.

11. Yilmaz Y: Review article: is non-alcoholic fatty liver disease a spectrum, or are steatosis and non-alcoholic steatohepatitis distinct conditions? Aliment Pharmacol Ther 2012, 36:815-823.

12. Sanyal AJ, Chalasani N, Kowdley KV, McCullough A, Diehl AM, Bass NM, Neuschwander-Tetri BA, Lavine JE, Tonascia J, Unalp A, Van Natta M, Clark J, Brunt EM, Kleiner DE, Hoofnagle JH, Robuck PR NASHCRN: Pioglitazone, vitamin E, or placebo for nonalcoholic steatohepatitis. New Engl J Med 2010, 362:1675-1685.

13. Chalasani N, Younossi Z, Lavine JE, Diehl AM, Brunt EM, Cusi K, Michael C, Arun Sanyal J: The diagnosis and management of non-alcoholic fatty liver disease: Practice Guideline by the American Association for the Study of Liver Diseases, American College of Gastroenterology, and the American Gastroenterological Association. Hepatology 2012, 55:2005-2023.

14. Chalasani N, Deeg MA, Crabb DW: Systemic levels of lipid peroxidation and its metabolic and dietary correlates in patients with nonalcoholic steatohepatitis. Am J Gastroenterol 2004, 99:1497-1502. 
15. Yesilova Z, Yaman H, Oktenli C, Ozcan A, Uygun A, Cakir E, Sanisoglu SY, Erdil A, Ates Y, Aslan M, Musabak U, Erbil MK, Karaeren N, Dagalp K: Systemic markers of lipid peroxidation and antioxidants in patients with nonalcoholic fatty liver disease. Am J Gastroenterol 2005, 100:850-855.

16. Narasimhan S, Gokulakrishnan K, Sampathkumar R, Farooq S, Ravikumar R, Mohan V, Balasubramanyam M: Oxidative stress is independently associated with non-alcoholic fatty liver disease (NAFLD) in subjects with and without type 2 diabetes. Clin Biochem 2010, 43:815-821.

17. Nobili V, Parola M, Alisi A, Marra F, Piemonte F, Mombello C, Sutti S, Povero $D$, Maina $V$, Novo E, Albano E: Oxidative stress parameters in paediatric non-alcoholic fatty liver disease. Intern J Molec Med 2010, 26:471-476.

18. Irie M, Sohda T, Iwata K, Kunimoto H, Fukunaga A, Kuno S, Yotsumoto K, Sakurai K, Iwashita H, Hirano G, Ueda SI, Yokoyama K, Morihara D, Nishizawa S, Anan A, Takeyama Y, Sakamoto M, Shakado S, Sakisaka S: Levels of the oxidative stress marker g-glutamyltranspeptidase at different stages of nonalcoholic fatty liver disease. J Intern Med Res 2012, 40:924-933.

19. Pirgon $\mathrm{O}$, Bilgin $\mathrm{H}$, Kurku H, Cekmez F, Dündar BN: Association between insulin resistance and oxidative stress parameters in obese adolescents with non-alcoholic fatty liver disease. J Clin Res Pediatr Endocrinol 2013, 5:33-39.

20. Bonnefont-Rousselot D, Ratziu V, Giral P, Charlotte F, Beucler I, Poynard T: For the lido study group: blood oxidative stress markers are unreliable markers of hepatic steatosis. Alim Pharmacol Ther 2006, 23:91-99.

21. Il'yasova D, Scarbrough P, Spasojevic I: Urinary biomarkers of oxidative status. Clin Chim Acta 2012, 413:1446-1453.

22. Montuschi P, Barnes PJ, Roberts LJ: Isoprostanes: markers and mediators of oxidative stress. FASEB J 2004, 18:1791-1800.

23. Praticò D: Prostanoid and isoprostanoid pathways in atherogenesis. Atherosclerosis 2008, 201:8-16.

24. Cave AC, Brewer AC, Narayanapanicker A, Ray R, Grieve DJ, Walker S, Shah AM: NADPH oxidases in cardiovascular health and disease. Antioxid Redox Signal 2006, 8:691-728.

25. Cangemi R, Angelico F, Loffredo L, Del Ben M, Pignatelli P, Martini A, Violi F: Oxidative stress-mediated arterial dysfunction in patients with metabolic syndrome: effect of ascorbic acid. Free Rad Biol Med 2007, 43:853-859.

26. Angelico F, Loffredo L, Pignatelli P, Augelletti T, Carnevale R, Pacella A, Albanese F, Mancini I, Di Santo S, Del Ben M, Violi F: Weight loss is associated with improved endothelial dysfunction via NOX2-generated oxidative stress down-regulation in patients with the metabolic syndrome. Int Emerg Med 2012, 7:219-227.

27. Loffredo L, Martino F, Carnevale R, Pignatelli P, Catasca E, Perri L, Calabrese CM, Palumbo MM, Baratta F, Del Ben M, Angelico F, Violi F: Obesity and hypercholesterolemia are associated with NOX-2 generated oxidative stress and arterial dysfunction. J Pediatr 2012, 161:1004-1009.

28. Del Ben M, Fabiani M, Loffredo L, Polimeni L, Carnevale R, Baratta F, Brunori M, Albanese F, Augelletti T, Violi F, Angelico F: Oxidative stress mediated arterial dysfunction in patients with obstructive sleep apnoea and the effect of continuous positive airway pressure treatment. BMC Pulm Med 2012, 23(12):36.

29. World Health Organisation: Definition diagnosis and classification of diabetes mellitus and its complications, Report of a WHO Consultation. Geneva, Switzerland: World Health Organisation; 1999.

30. Grundy SM, Cleeman Jl, Daniels SR, Donato KA, Eckel RH, Franklin BA, Gordon DJ, Krauss RM, Savage PJ, Smith SC Jr, Spertus JA, Costa F, American Heart Association; National Heart, Lung, and Blood Institute: Diagnosis and management of the metabolic syndrome: an american heart association/national heart, lung, and blood institute scientific statement. Circulation 2005, 112:2735-2752.

31. Matthews DR, Hosker JP, Rudenski AS, Naylor BA, Treacher DF, Turner RC: Homeostasis model assessment: insulin resistance and beta-cell function from fasting plasma glucose and insulin concentrations in man. Diabetologia 1985, 28:412-419.

32. Pignatelli P, Carnevale R, Cangemi R, Loffredo L, Sanguigni V, Stefanutti C, Basili S, Violi F: Atorvastatin inhibits gp91phox circulating levels in patients with hypercholesterolemia. Arterioscler Thromb Vasc Biol 2010, 30:360-367.

33. Wang Z, Ciabattoni G, Creminon C, Lawson J, Fitzgerald GA, Patrono C, Maclouf J: Immunological characterization of urinary 8-epi-prostaglandin F2 alpha excretion in man. J Pharmacol Exper Ther 1995, 275:94-100.

34. Hamaguchi M, Kojima T, Itoh Y, Harano Y, Fujii K, Nakajima T, Kato T, Takeda N, Okuda J, Ida K, Kawahito Y, Yoshikawa T, Okanoue T: The severity of ultrasound findings in nonalcoholic fatty liver disease reflects the metabolic syndrome and visceral fat accumulation. Am J Gastroenterol 2007, 102:1-8

35. Seki S, Kitata T, Yamada T, Sakaguchi H, Nakatami K, Wakasa K: In situ detection of lipid peroxidation and oxidative DNA damage in non-alcoholic fatty liver diseases. J Hepatol 2002, 37:56-62.

36. Roest M, Voorbij HA, Van der Schouw YT, Peeters PH, Teerlink T, Scheffer PG: High levels of urinary F2-isoprostanes predict cardiovascular mortality in postmenopausal women. J Clin Lipidol 2008, 2:298-303.

37. Feldstein AE, Anna Wieckowska A, Rocio Lopez A, Yao-Chang L, Nizar Zein N, Arthur McCullough J, Rocio Lopez A, Yao-Chang L, Nizar N, Arthur J: Cytokeratin-18 fragment levels as noninvasive biomarkers for nonalcoholic steatohepatitis: a multicenter validation study. Hepatology 2009, 50:1072-1078.

38. Dasarathy S, Dasarathy J, Khiyami A, Joseph R, Lopez R, McCullough AJ: Validity of real time ultrasound in the diagnosis of hepatic steatosis: a prospective study. J Hepatol 2009, 51:1061-1067.

doi:10.1186/1471-230X-14-81

Cite this article as: Del Ben et al:: NOX2-generated oxidative stress is associated with severity of ultrasound liver steatosis in patients with non-alcoholic fatty liver disease. BMC Gastroenterology 2014 14:81.

\section{Submit your next manuscript to BioMed Central and take full advantage of:}

- Convenient online submission

- Thorough peer review

- No space constraints or color figure charges

- Immediate publication on acceptance

- Inclusion in PubMed, CAS, Scopus and Google Scholar

- Research which is freely available for redistribution

Submit your manuscript at www.biomedcentral.com/submit
C) Biomed Central 